\title{
Compact orthogonal-dispersion device using a prism and a transmission grating
}

Qinghua Yang ${ }^{1 *}$ and Weiqiang Wang ${ }^{2}$

\begin{abstract}
Background: Spectroscopy was one of the main applications of gratings. Most spectrometers today are based on reflection grating designs, since high quality transmission grating technologies have only matured over the last several years. Conventional cross-dispersion systems are based on the reflection grating. For traditional direct-vision or constant-dispersion grism, both the prism and grating have the same dispersion direction.

Methods: This paper presents an orthogonal-dispersion device based on a transmission grating attached to a prism. The dispersion directions of the prism and transmission grating are orthogonal. The analytical expressions for the dispersion of this device are derived in detail. The numerical results and ray-tracing simulations by ZEMAX software are shown.

Results: The simulation results show that the spectrum is spread over 5 diffraction orders, $m=3$ to -1 , the non-diffracted order $m=0$ is straight, and the other orders are curved.

Conclusions: The device will be able to provide a compact, small-sized and broadband orthogonal- dispersion device that is suitable for the medium resolution spectrometer.
\end{abstract}

Keywords: Orthogonal dispersion, Transmission grating, Prism, Spectrometer

\section{Background}

Spectroscopy was one of the earliest applications of gratings, and it remains one of the main grating uses [1]. More particularly, the echelle grating suggested first by Harrison [2] is preferred in a number of spectrometers. The echelle grating is usually used in high diffraction orders that will overlap. To solve this problem, traditional echelle spectrometers usually employ cut-off filters or a cross disperser in the form of low-dispersion gratings or prisms. An early echelle spectrometer uses two planar reflection gratings, i.e., an echelle grating and a reflection grating, operating in two perpendicular planes [3]. The resulting dispersion produces a two-dimensional spectrogram including several lines side by side that correspond to different diffraction orders, namely, several diffraction-order lines side by side with the wavelength varying with its position along each line. As an improved echelle spectrometer [4], the second grating is replaced by a combination of a reflection grating with a prism so that the light from the echelle grating is

\footnotetext{
* Correspondence: yangqh812@126.com

'School of Physics and Optoelectronic Engineering, Xidian University, Xi'an

710071, China

Full list of author information is available at the end of the article
}

refracted into the prism, diffracted by the grating, then refracted back out of the prism. The diffraction orders can be spread out almost evenly by choosing the prism parameters, allowing more of the detector area to record useful information. For the above referenced echelle spectrometers, both the incident and diffracted light rays are perpendicular to the grating grooves.

The diffraction grating can also be used in the conical diffraction mode [5-10] in which the incident light beam is not perpendicular to the grating grooves, thus the diffracted light rays lie on a circular cone rather than in a plane. Typically, the conical diffraction spectrometers reported in [11] uses a Littrow configuration coupled with a shared focusing lens and a linear detector array, and they are compact with a substantially reduced size. Nevertheless, these two designs are unable to separate the overlapping diffraction orders of the echelle grating. On the contrary, the Littrow conical diffraction spectrometer proposed by [12] includes an additional prism for separating the overlapping diffraction orders of the echelle grating to produce two-dimensional high dispersion without requiring a large field of view. 
Most spectrometers today are based on reflection grating designs [13-15], since high quality transmission grating technologies have only matured over the last several years. However, it is currently possible to produce high quality transmission gratings that rival reflection gratings in all aspects. Accordingly, transmission gratings can be used as dispersive devices in the infrared, visible or near-ultraviolet light [16-21]. Transmission gratings offer low alignment sensitivity, which helps minimize alignment errors. A transmission grating offers a basic simplicity for optical designs that can be beneficial in spectral separation. In certain types of instruments, transmission gratings are much more convenient to use than reflection gratings. For example, a configuration inserts a transmission grating in front of the lens, which can be used for studying the composition of falling meteors or the reentry of space vehicles, where the distant luminous streak becomes the entrance slit.

The direct-vision grism combines a low-dispersion transmission grating with a beam-bending prism to provide in-line viewing for a single wavelength [22]. The purpose of the prism is simply to change the direction of a given diffracted wavelength, for zero net deviation. The constant-dispersion grism consists of a prism and a transmission grating to disperse the spectrum so that the spectrum fringes are equally spaced across the detector. Namely, for the constantdispersion grism the angular dispersion with respect to wave number is approximately constant. Both the transmission grating and prism contribute to the dispersion, and the net deviation angles are not constrained to be zero. For the direct-vision or constantdispersion grism, both the prism and transmission grating have the same dispersion direction.

This paper is a theoretical study of a compact, smallsized and broadband orthogonal-dispersion prismgrating device used for spectrometers. Section 2 presents the basic principle and derives the analytical expressions for the dispersion of the device. Section 3 shows the numerical results and ray-tracing simulations by ZEMAX software. The last section gives the conclusion.

\section{Methods}

Figure 1a shows the optical layout of the orthogonaldispersion prism-grating device $(P G D)$ that combines a prism with a transmission grating. The dispersion directions of the prism and transmission grating are orthogonal. The transmission grating is superimposed on the hypotenuse surface of an optical wedge, and the right-angle surface of the optical wedge is directly attached to one surface of the prism. The grating normal is parallel to $y$-axis, the grating grooves are parallel to z-axis, and the $y^{\prime}$-axis is perpendicular to the right-angle surface of the optical wedge. The prism disperses the incident light along the vertical direction (i.e., $\mathrm{z}$-axis), then the transmission grating disperses the separated beams along the horizontal direction (i.e., $\mathrm{x}$-axis). The spectrum is spread out in two dimensions.

Suppose that $\gamma$ denotes the vertex angle of the prism, $\gamma_{1}$ denotes the vertex angle of the optical wedge, $n_{p}\left(\lambda_{i}\right)$ is the refractive index of the prism for wavelength $\lambda_{i}$, and $n_{g}\left(\lambda_{i}\right)$ is the refractive index of the grating material for wavelength $\lambda_{i}$.

Figure $1 \mathrm{~b}$ shows the ray tracing in the sagittal plane (i.e., $x^{\prime}-y^{\prime}$ plane), in which $\delta$ is the angle between the inward normal vector of the grating and the projection of the incident wave vector $\vec{k}$ onto the normal plane of the grating (i.e., $x-y$ plane). It is easily obtained that $\delta=\gamma_{1}$.

Figure 1c depicts the ray tracing in the meridian plane (i.e., $\mathrm{y}^{\prime}-\mathrm{z}^{\prime}$ plane), in which $\varepsilon$ is the angle between the incident wave vector $\vec{k}$ and the normal plane of the grating. A ray enters the prism at angle $\theta$, is refracted at angle $\theta_{1}$, leaves the prism at angle $\theta_{2}$, and is refracted into the grating material at angle $\theta_{3}$. It is easily obtained that

$$
\begin{aligned}
& \sin \theta=n_{p}\left(\lambda_{i}\right) \sin \theta_{1}, \\
& n_{p}\left(\lambda_{i}\right) \sin \theta_{2}=n_{g}\left(\lambda_{1}\right) \sin \theta_{3}, \\
& \varepsilon=\theta_{3}, \\
& \theta_{1}+\theta_{2}=\gamma
\end{aligned}
$$

From Eq. (1a-d), the angle $\varepsilon$ is given by

$$
\begin{aligned}
n_{g}\left(\lambda_{i}\right) \sin \varepsilon & =n_{p}\left(\lambda_{i}\right) \sin \left(\gamma-\theta_{1}\right) \\
& =n_{p}\left(\lambda_{i}\right)\left(\sin \gamma \cos \theta_{1}-\cos \gamma \sin \theta_{1}\right) \\
& =n_{p}\left(\lambda_{i}\right)\left(\sin \gamma \sqrt{1-\frac{\sin ^{2} \theta}{n_{p}^{2}\left(\lambda_{i}\right)}}-\frac{\cos \gamma \sin \theta}{n_{p}\left(\lambda_{i}\right)}\right) \\
& =\sin \gamma \sqrt{n_{p}^{2}\left(\lambda_{i}\right)-\sin ^{2} \theta}-\cos \gamma \sin \theta .
\end{aligned}
$$

Let the plane of incidence be the plane that is made up of the incident light ray and the grating normal. Let the normal plane of the grating be the plane that is perpendicular to the grating grooves. In the conical diffraction case, the grating equation of a plane transmission grating is given by [1].

$$
m \lambda_{i}=d\left[n_{g}\left(\lambda_{i}\right) \sin \alpha-\sin \beta_{m}\right] \cos \varepsilon
$$

where $m$ is the diffraction order, $\lambda_{i}$ is the wavelength of light, $d$ is the groove spacing of grating, $\varepsilon$ is the angle between the incident light path and the plane perpendicular to the grating grooves, $\alpha$ is the 

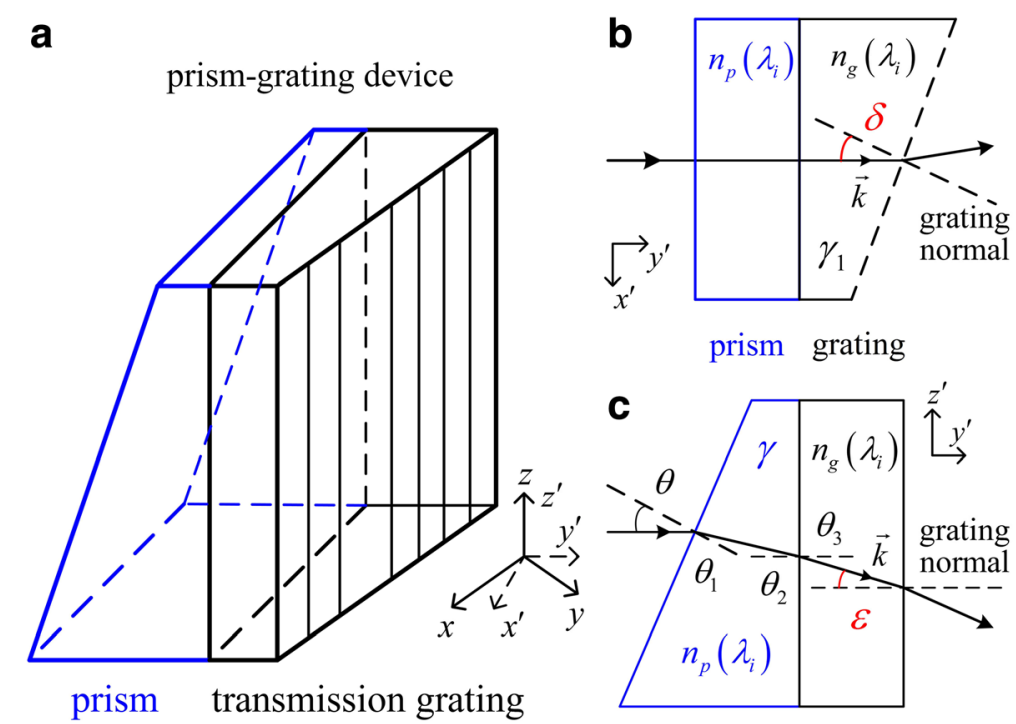

Fig. 1 a Optical layout of the prism-grating device, in which the prism disperses the incident light along $z$-axis, the grating normal is parallel to $y$-axis, and the grating grooves are parallel to $z$-axis. b Ray tracing in the sagittal plane (i.e., $x^{\prime}-y^{\prime}$ plane). c Ray tracing in the meridian plane (i.e., $y^{\prime}-z^{\prime}$ plane)

angle of incidence measured from the grating normal, $\beta_{m}$ is the diffraction angle measured from the grating normal. The angle sign convention is that angles measured counter-clockwise from the normal are positive and angles measured clockwise from the normal are negative.
Figure 2(a) shows the conical diffraction by a plane transmission grating, in which the grating lies in the $\mathrm{x}-\mathrm{z}$ plane, the grating grooves are parallel to $\mathrm{z}$-axis, and the grating normal is parallel to $y$-axis. Both the incident ray and the reflected rays lie in the $y<0$ half-space; the transmitted diffracted rays lie in the
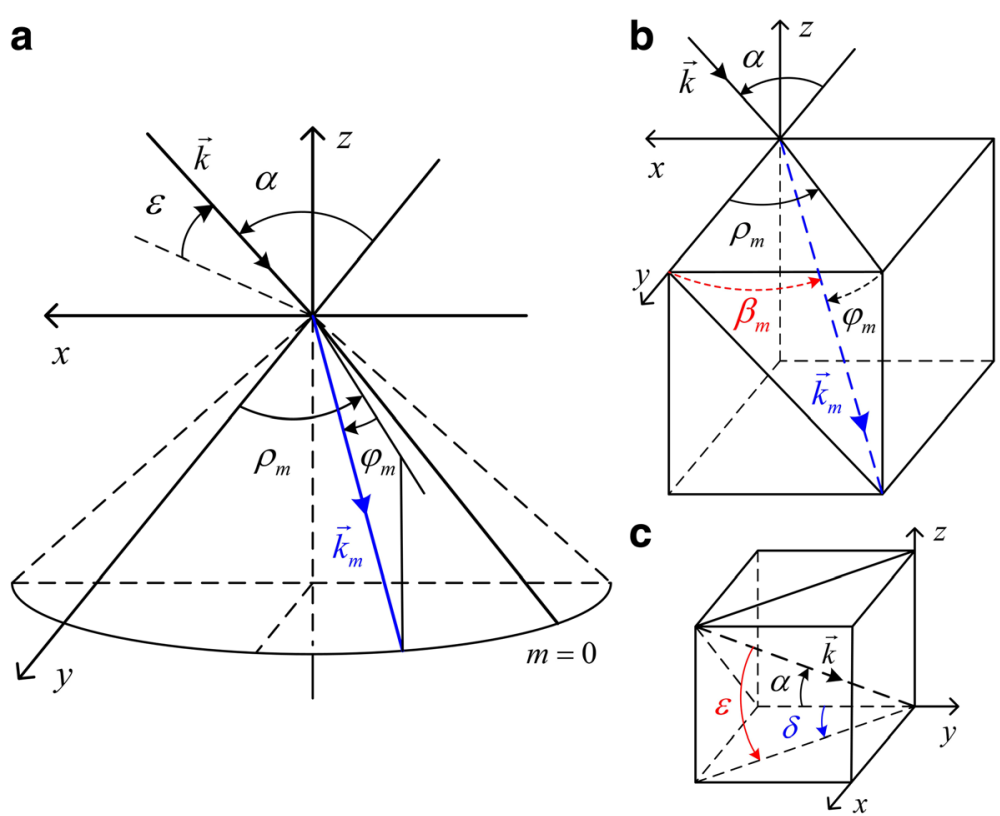

Fig. 2 a Conical diffraction by a plane transmission grating, where a rectangular coordinate system is assumed with the $z$-axis parallel to the grating grooves and the $y$-axis parallel to the grating normal. $\mathbf{b}$ Geometry diagram of a diffracted light ray. c Geometry diagram of an incident wave vector 


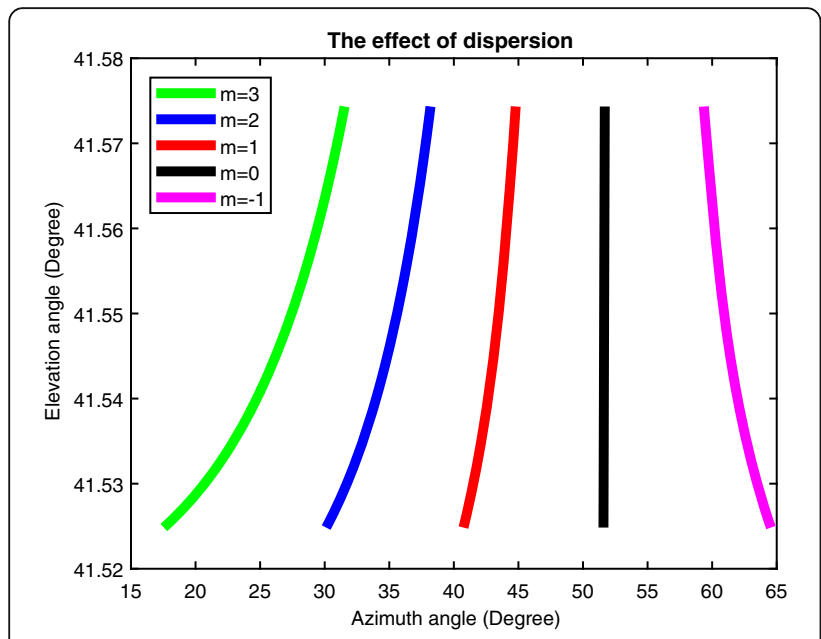

Fig. 3 The effect of dispersion for a prism-grating device (for source spectrum $500 \mathrm{~nm}-800 \mathrm{~nm}$ )

$y>0$ half-spaceThat is, the incident light is dispersed on the opposite side of the transmission grating. All diffracted rays fall on the surface of a half-cone whose cone axis is parallel to the grating grooves.

Figure 2(b) shows the geometry diagram of a diffracted light ray. The direction of the diffracted ray with a wave vector $\vec{k}_{m}=k_{m x} \vec{e}_{x}+k_{m y} \vec{e}_{y}+k_{m z} \vec{e}_{z}$ is given by two parameters: (1) $\phi_{m}$ is the angle between the diffracted ray and the normal plane of the grating and (2) $\rho_{m}$ is the angle between the outward normal vector of the grating and the projection of the diffracted ray onto the normal plane of the grating. We can get

$$
\sin \beta_{m}=\sqrt{k_{m x}^{2}+k_{m z}^{2}} / k_{m}
$$

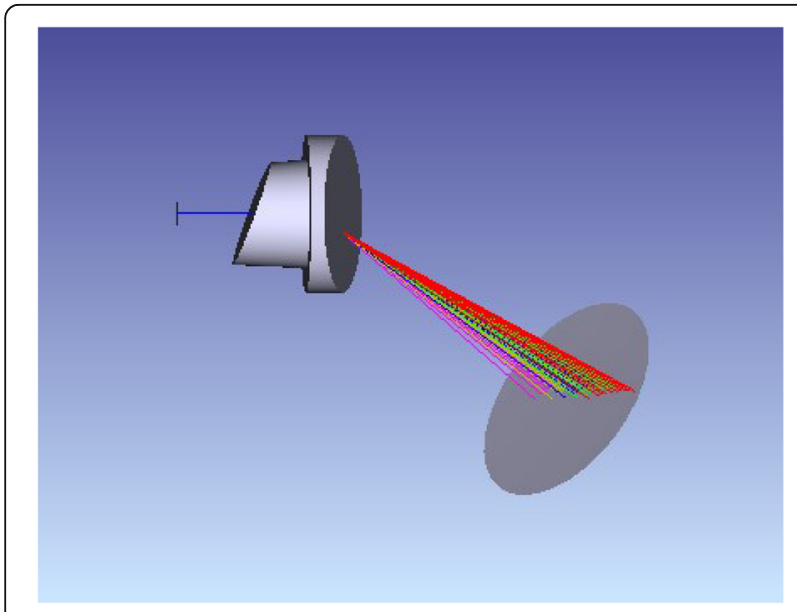

Fig. 4 Shaded Model 1 by ZEMAX

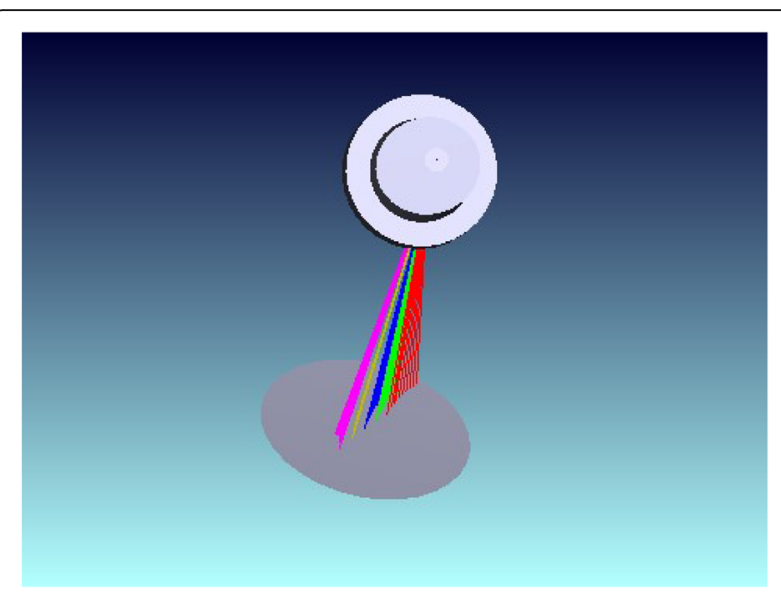

Fig. 5 Shaded Model 2 by ZEMAX with Color Rays representing the different Diffraction orders

$$
\begin{aligned}
& \sin \rho_{m}=k_{m x} / \sqrt{k_{m x}^{2}+k_{m y}^{2}}, \\
& \cos \phi_{m}=\sqrt{k_{m x}^{2}+k_{m y}^{2}} / k_{m}, \\
& \sin \phi_{m}=k_{m z} / k_{m}
\end{aligned}
$$

Based on Eqs. (4a-d), it can be obtained that

$$
\sin \rho_{m}=\sqrt{\frac{\sin ^{2} \beta_{m}-\sin ^{2} \phi_{m}}{1-\sin ^{2} \phi_{m}}}
$$

Figure 2(c) shows the geometry diagram of an incident wave vector. A light ray, with a wave vector $\vec{k}=k_{x} \vec{e}_{x}$ $+k_{y} \vec{e}_{y}+k_{z} \vec{e}_{z}$ and its modulus $k=2 \pi / \lambda$, is incident on the grating at an off-plane direction $(\varepsilon \neq 0)$. We can obtain

$$
\begin{aligned}
& \tan \delta=k_{x} / k_{y}, \\
& \cos \alpha=k_{y} / k, \\
& \sin \varepsilon=k_{z} / k, \\
& k^{2}=k_{x}^{2}+k_{y}^{2}+k_{z}^{2}
\end{aligned}
$$

From Eqs. (6a-d), it can be obtained that

$$
\begin{aligned}
\sin \alpha & =\sqrt{\sin ^{2} \delta+\cos ^{2} \delta \sin ^{2} \varepsilon} \\
& =\sqrt{\sin ^{2} \gamma_{1}+\cos ^{2} \gamma_{1} \sin ^{2} \varepsilon}
\end{aligned}
$$

According to the law of refraction and the geometry, we can get

$$
\sin \phi_{m}=n_{g}\left(\lambda_{i}\right) \sin \varepsilon
$$

Based on Eqs. (2) and (8), the elevation angle $\phi_{m}$, for the PGD, is given by 


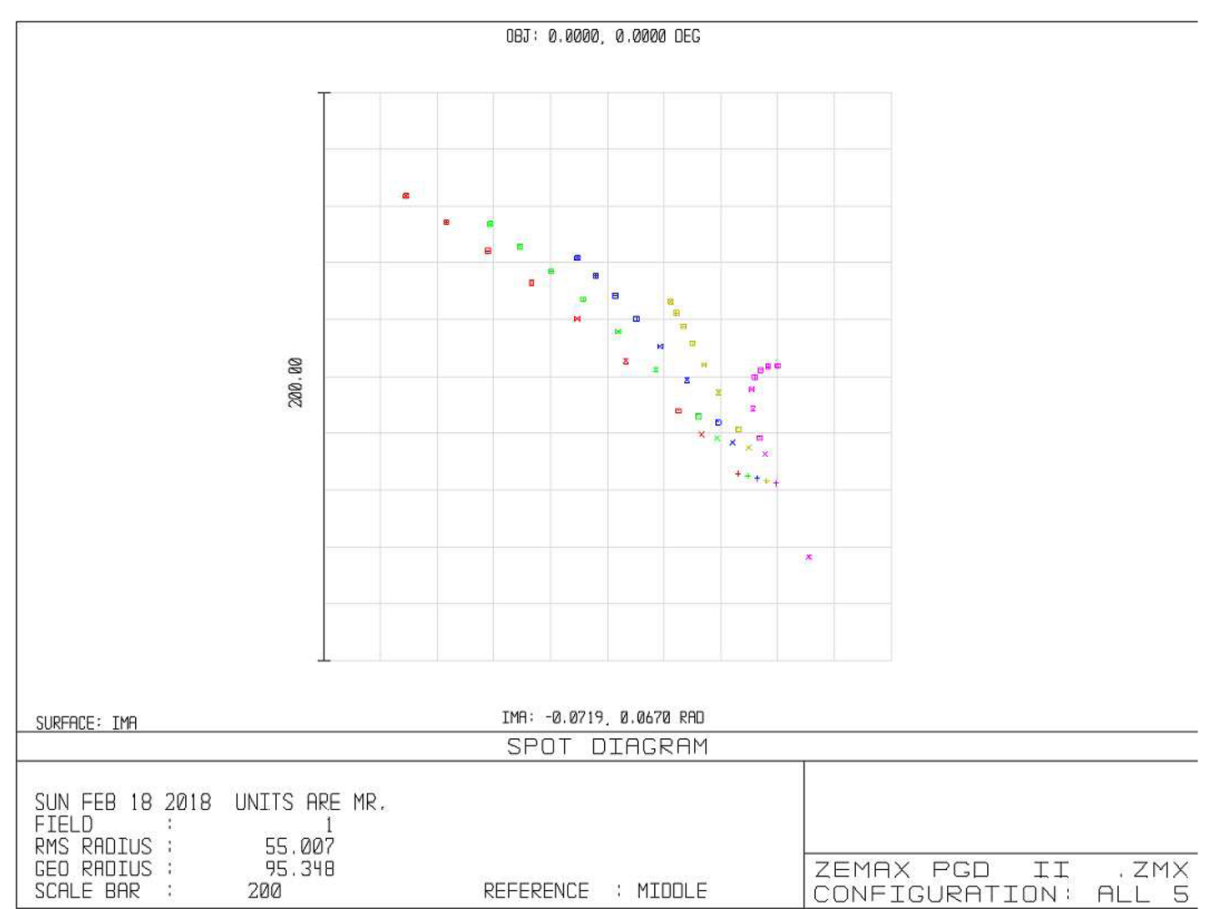

Fig. 6 Spot Diagram 1 with Color Rays representing the different Diffraction orders

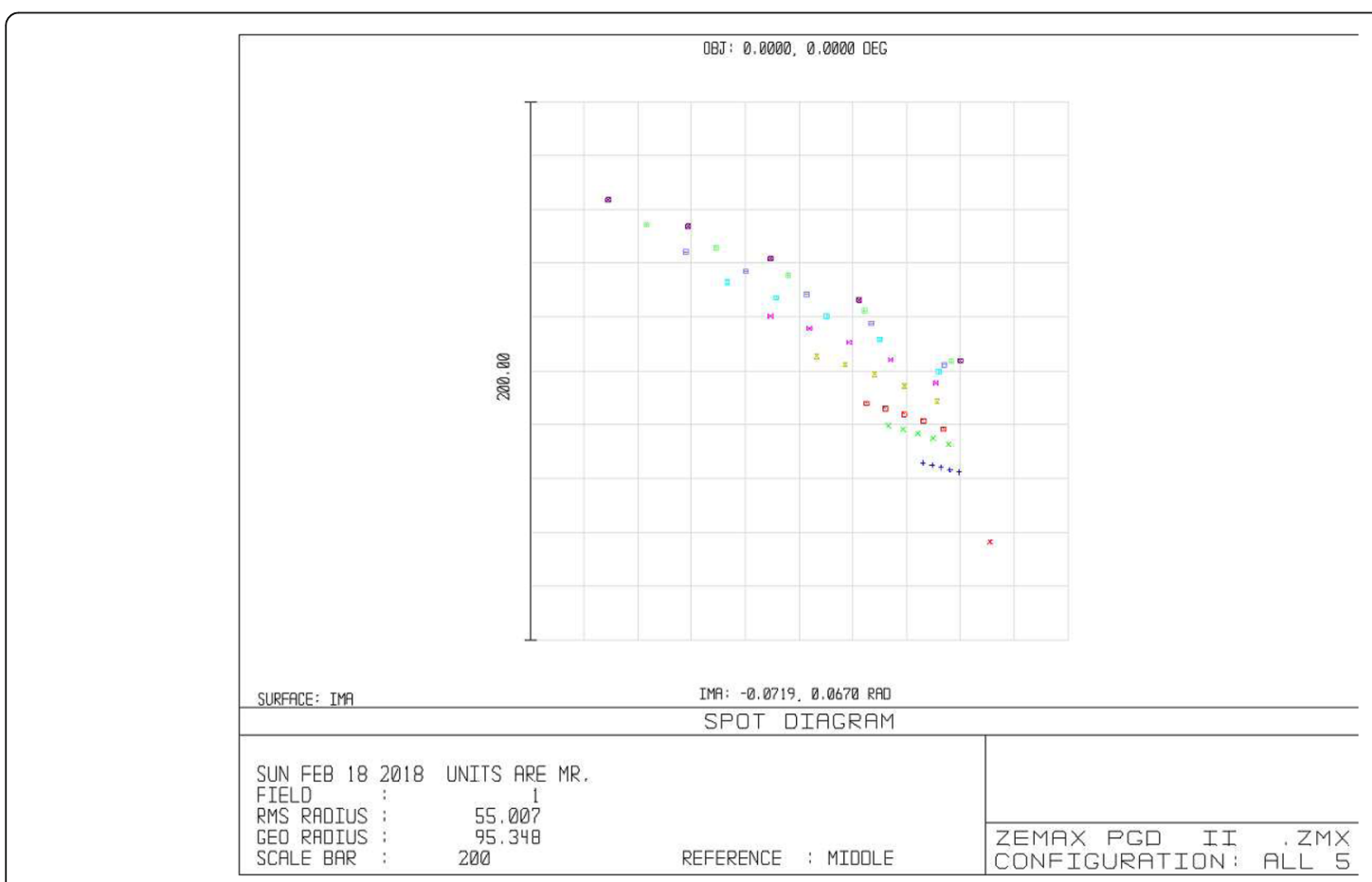

Fig. 7 Spot Diagram 2 with Color Rays representing the different Wavelengths 


$$
\sin \phi_{m}=\sin \gamma \sqrt{n_{p}^{2}\left(\lambda_{i}\right)-\sin ^{2} \theta}-\cos \gamma \sin \theta
$$

Based on Eqs. (3), (5), (7) and (8), the azimuth angle $\rho_{m}$, for the PGD, is given by

$$
\begin{aligned}
\sin \rho_{m} & =\sqrt{\frac{\sin ^{2} \beta_{m}-\sin ^{2} \phi_{m}}{1-\sin ^{2} \phi_{m}}} \\
& =\sqrt{\frac{\left(\frac{m l_{i}}{d \cos \varepsilon}-n_{g}\left(\lambda_{i}\right) \sin \alpha\right)^{2}-\sin ^{2} \phi_{m}}{1-\sin ^{2} \phi_{m}}} \\
& =\sqrt{\frac{\left(\frac{m l_{i}}{d \cos \varepsilon}-n_{g}\left(\lambda_{i}\right) \sqrt{\sin ^{2} \gamma_{1}+\cos ^{2} \gamma_{1} \sin ^{2} \varepsilon}\right)^{2}-\sin ^{2} \phi_{m}}{1-\sin ^{2} \phi_{m}}} \\
& =\sqrt{\frac{\left(\frac{m \lambda_{1}}{d \sqrt{1-\frac{\sin ^{2} \phi_{m}}{n_{g}\left(\lambda_{i}\right)}}}-n_{g}\left(\lambda_{i}\right) \sqrt{\sin ^{2} \gamma_{1}}+\cos ^{2} \gamma_{1} \frac{\sin ^{2} \phi_{m} n_{g}^{2}\left(\lambda_{i}\right)}{1}\right)^{2}-\sin ^{2} \phi_{m}}{1-\sin ^{2} \phi_{m}}} \\
& =\sqrt{\frac{\left(\frac{n_{g}\left(\lambda_{i}\right) m \lambda_{i}}{d \sqrt{n_{g}^{2}\left(\lambda_{i}\right)-\sin ^{2} \phi_{m}}}-\sqrt{n_{g}^{2}\left(\lambda_{i}\right) \sin ^{2} \gamma_{1}+\cos ^{2} \gamma_{1} \sin ^{2} \phi_{m}}\right)^{2}-\sin ^{2} \phi_{m}}{1-\sin ^{2} \phi_{m}}}
\end{aligned}
$$

Eqs. (9) and (10) completely specify the diffracted ray angles in terms of the incident ray angles. For an incident ray of a given wavelength, the diffracted rays will all have the same value of $\phi_{m}$ given in Eq. (9) and will therefore all lie in a common conical surface, as shown in Fig. 2(a). For reference, if $\varepsilon=0$, the conical diffraction grating equation [Eq. (3)] simplifies to yield the classical in-plane diffraction grating equation, $m \lambda_{i}=d\left[n_{g}\left(\lambda_{i}\right) \sin \alpha-\sin \beta_{m}\right]$, for a plane transmission grating.

\section{Results and discussion}

For a source spectrum with a spectral range from $500 \mathrm{~nm}$ to $800 \mathrm{~nm}$, for example, we can consider a transmission grating with 75 grooves $/ \mathrm{mm}$ and a prism that is made of Zinc sulfide ( $\mathrm{ZnS}$ ) with a refractive index formula as [23].

$$
\mathrm{n}^{2}(\lambda)=8.393+\frac{0.14383}{\lambda^{2}-0.2421^{2}}+\frac{4430.99}{\lambda^{2}-36.71^{2}}
$$

Suppose that the vertex angle of the prism is $\gamma=20^{\circ}$, the angle of incidence on the prism is $\theta=20^{\circ}$, and the vertex angle of the optical wedge is $\gamma_{1}=12^{\circ}$. According to Eqs. (9)-(11), the separation of the spectral orders is illustrated in Fig. 3, in which the spectrum is spread over 5 diffraction orders, $m=3$ to -1 , with the azimuth angle from small to big between 15 and 65 degrees. Moreover, the non-diffracted order $m=0$ is straight, but the other orders are curved.
Suppose that the entrance pupil diameter is $10 \mathrm{~mm}$, the center thickness of the prism is $22 \mathrm{~mm}$, the center thickness of the grating is $15 \mathrm{~mm}$. For a wavelength range from $500 \mathrm{~nm}$ to $900 \mathrm{~nm}$ in diffraction order - 1 through 3, the ray-tracing simulations by ZEMAX software for the orthogonal-dispersion PGD are shown in the following several figures. Figure 4 shows one Shaded Model, Fig. 5 shows the other Shaded Model in which the different color rays represent the different diffraction orders, Fig. 6 shows one Spot Diagram in which the different color rays represent the different diffraction orders, and Fig. 7 shows another Spot Diagram in which the different color rays represent the different wavelengths. According to Figs. 6 and 7, the non-diffracted order $m=0$ is straight, but the other orders are curved especially in the -1 order.

\section{Conclusions}

An orthogonal-dispersion PGD was investigated in theory. The ray-tracing simulations by ZEMAX indicated the accuracy of the theoretical analysis and mathematical formulas. In order to improve the spectral resolution, the dispersive power of the PGD needs to be improved, which can be realized by either appropriately increasing the vertex angle of the prism, properly reducing the groove spacing of grating, or both. In addition, the dispersive power of the PGD can also be changed by choosing different materials of the prism and grating. There are two main features to the PGD. First, the orthogonal-dispersion PGD is based on the transmission grating, whereas traditional cross-dispersion systems are based on the reflection grating. Second, the transmission grating is superimposed on the hypotenuse surface of an optical wedge with a right-angle surface directly attached to the prism, which not only makes the PGD compact, but also makes the air-glass interfaces fewer and therefore makes the reflection loss less. The unique design makes the PGD capable of providing a compact, smallsized and broadband orthogonal-dispersion device that is applicable to the medium resolution spectrometers.

Abbreviations

PGD: Prism-grating device

Acknowledgements

None.

Funding

This work was supported by the National Natural Science Foundation of China (NSFC) (Grant number 61605151).

Availability of data and materials

The data and materials supporting the conclusions of this article is included within the article.

Authors' contributions

QY has come up with the idea, derived the formulas, and performed the ray-tracing simulations by ZEMAX software. QY was the First Author and 
Corresponding Author. WW performed numerical results. Both authors read and approved the final manuscript.

\section{Competing interests}

The authors declare that they have no competing interests.

\section{Publisher's Note}

Springer Nature remains neutral with regard to jurisdictional claims in published maps and institutional affiliations.

\section{Author details}

'School of Physics and Optoelectronic Engineering, Xidian University, Xi'an 710071, China. ${ }^{2}$ State Key Laboratory of Transient Optics and Photonics, Xi'an Institute of Optics and Precision Mechanics, Chinese Academy of Sciences (CAS), Xi'an 710119, China.

Received: 6 January 2018 Accepted: 4 March 2018

Published online: 12 March 2018

\section{References}

1. Palmer, C., Loewen, E.: Diffraction Grating Handbook. Newport Corporation, New York (2005)

2. Harrison, G.R.: The production of diffraction gratings: II. The design of echelle gratings and spectrographs. J. Opt. Soc. Am. 39(7), 522-527 (1949)

3. Dantzler, A.A.: Echelle spectrograph software design aid. Appl. Opt. 24(24), 4504-4508 (1985)

4. Dantzler, A.A.: Echelle grism spectrograph. Appl. Opt. 25(23), 4267-4287 (1986)

5. Cash, W.: Echelle spectrographs at grazing incidence. Appl. Opt. 21(4), 710-717 (1982)

6. Cornet, P., Chandezon, J., Faure, C.: Conical diffraction of a plane wave by an inclined parallel-plate grating. J. Opt. Soc. Am. A. 14(2), 437-449 (1997)

7. Goray, L.I., Schmidt, G.: Solving conical diffraction grating problems with integral equations. J. Opt. Soc. Am. A. 27(3), 585-597 (2010)

8. Werner, W.: X-ray and extended UV spectrometer designs based on offplane grating mountings. Space Sci. Rev. 29(4), 455-459 (1981)

9. Werner, W.: Comparison of various grazing incidence spectrometer designs based on conical diffraction. Appl. Opt. 23(14), 2408-2411 (1984)

10. Lemaire, P.: Ultraviolet conical diffraction: a near-stigmatic tandem grating mounting spectrometer. Appl. Opt. 30(10), 1294-1302 (1991)

11. Everett, M.J., Zhou, Y., Horn, J.M.M., O'Hara, K., Foley, J.P.: Littrow spectrometer and a spectral domain optical coherence tomography system with a Littrow spectrometer. US patent 7456957 B2, United States (2008)

12. Yang, Q.: Compact high-resolution Littrow conical diffraction spectrometer. Appl. Opt. 55(18), 4801-4807 (2016)

13. Ballester, P., Rosa, M.R.: Modeling echelle spectrographs. Astron. Astrophys. Suppl. Ser. 126(3), 563-571 (1997)

14. Ge, J., Angel, J.R.P., Jacobsen, B., Woolf, N., Fugate, R.Q., Black, J.H., Lloyd-Hart, M.: An optical ultra-high-resolution cross-dispersed echelle spectrograph with adaptive optics. Publ. Astron. Soc. Pac. 114(798), 879-891 (2002)

15. Yin, L., Bayanheshig, Yang, J., Lu, Y., Zhang, R., Sun, C., Cui, J.: High-accuracy spectral reduction algorithm for the échelle spectrometer. Appl. Opt. 55(13) 3574-3581 (2016)

16. Yang, J., Ding, Y., Zhang, W., Zhang, J., Zheng, Z.: Precise measurement technology of soft-x-ray spectrum using dual transmission grating spectrometer. Rev. Sci. Instrum. 74(10), 4268-4272 (2003)

17. Weisberg, A., Craparo, J., De Saro, R., Pawluczyk, R.: Comparison of a transmission grating spectrometer to a reflective grating spectrometer for standoff laser-induced breakdown spectroscopy measurements. Appl. Opt. 49(13), C200-C210 (2010)

18. Wang, C., Ding, Z., Mei, S., Yu, H., Hong, W., Yan, Y., Shen, W.: Ultralong-range phase imaging with orthogonal dispersive spectral-domain optical coherence tomography. Opt. Lett. 37(21), 4555-4557 (2012)

19. Bao, W., Ding, Z., Li, P., Chen, Z., Shen, Y., Wang, C.: Orthogonal dispersive spectral-domain optical coherence tomography. Opt. Express. 22(8), 10081-10090 (2014)

20. $\mathrm{Xu}, \mathrm{K}$ : Integrated silicon directly modulated light source using p-well in standard CMOS technology. IEEE Sensors J. 16(16), 6184-6191 (2016)
21. Xu, K., Li, G.P.: A novel way to improve the quantum efficiency of silicon light-emitting diode in a standard silicon complementary metal-oxidesemiconductor technology. J. Appl. Phys. 113(10), 103106 (2013)

22. Schroeder, D.J.: Astronomical optics. Academic, San Diego (1987)

23. Bass, M., Li, G., Stryland, E.V.: Handbook of Optics. McGraw-Hill, New York (2010)

\section{Submit your manuscript to a SpringerOpen ${ }^{\circ}$ journal and benefit from:}

- Convenient online submission

- Rigorous peer review

- Open access: articles freely available online

- High visibility within the field

Retaining the copyright to your article

Submit your next manuscript at $\boldsymbol{\sim}$ springeropen.com 